\title{
Modelo para otimização hidroenergética da operação de bombeamento em sistemas de distribuição de água
}

\author{
Hydro energy optimization model of pumping operation in water distribution systems
}

Anselmo Claudino de Sousa' ${ }^{\circledR}$, Alexandre Kepler Soares ${ }^{2 *}$ (1)

\section{RESUMO}

Neste trabalho foi desenvolvido um modelo multiobjetivo para otimização da operação de sistemas de distribuição de água (SDAs), visando alcançar a eficiência hidroenergética considerando três objetivos: redução das perdas por vazamento; redução do custo de energia elétrica no bombeamento; e maximização da confiabilidade do sistema. O modelo de otimização foi concebido pela implementação de uma rotina computacional entre os algoritmos genéticos NSGAll e SPEA e o simulador hidráulico EPANET. O modelo foi aplicado a um SDA hipotético e demonstrou ser adequado para gerar um conjunto ótimo de regras operacionais. Dentre as soluções geradas pelos dois algoritmos, constatou-se que a diminuição do custo de energia elétrica no bombeamento não implicou, necessariamente, redução das perdas por vazamentos, ou seja, os dois objetivos podem ser conflitantes em SDAs caracterizados pela presença de reservatórios internos.

Palavras-chave: sistemas de distribuição de água; eficiência hidroenergética; algoritmos evolucionários.

\begin{abstract}
In this work, a multi-objective model for operational optimization of water distribution systems has been developed in order to achieve hydro energy efficiency. Three objectives were considered: minimizing of leakage, minimizing of pumping energy costs, and maximizing system reliability. The optimization model was conceived by the implementation of a computational routine between a dyhraulic simulator (EPANET) and by genetic algorithms NSGAll and SPEA. The proposed model has been applied to the optimization of a hypothetical water distribution network to generate an optimal set of operational rules. Some solutions obtained by the two algorithms showed that a decrease in pumping energy costs did not necessarily imply in leakage reduction. This means that both objectives can be conflicting in water distribution systems with internal tanks.
\end{abstract}

Keywords: water distribution systems; hydro energy efficiency; evolutionary algorithms.

\section{INTRODUÇÃO}

A população mundial vem se consolidando como uma sociedade urbana. No Brasil, 84,4\% da população reside em cidades (IBGE, 2011), o que contribui para aumentar a demanda pelos serviços de abastecimento de água. Diante da demanda crescente por água potável, torna-se necessário o aumento da eficiência dos sistemas de distribuição de água (SDAs). Entretanto, dados do Diagnóstico dos Serviços de Água e Esgoto (SNIS, 2018) demonstram ainda ineficiência dos SDAs brasileiros, visto que o valor médio das perdas de água durante a distribuição é de 38,5\%. Parte dessas perdas ocorre devido aos vazamentos de água ao longo da rede de distribuição.

A operação eficiente de um SDA pode reduzir os índices de perda de água por vazamento e promover a redução do consumo de energia elétrica a partir de técnicas de otimização hidroenergética. Pesquisas realizadas por Colombo e Karney $(2002 ; 2005)$ sugerem que os vazamentos existentes podem ser relacionados com o aumento do consumo de energia elétrica. Gonçalves et al. (2009) apontam que durante a ocorrência de vazamentos há, também, desperdício de outros insumos, como energia elétrica, observando a influência das perdas reais no consumo de energia, pois quanto maior o volume de vazamentos, maior será a vazão bombeada, requerendo o incremento de potência fornecida ao sistema. Estima-se que entre 2 e 3\% do consumo de energia elétrica no Brasil ocorra no bombeamento de água até as residências (GOMES; CARVALHO, 2012). Um quarto dessa energia poderia ser poupado se as redes e os sistemas de bombeamento de água fossem mais eficientes (JAMES; CAMPBELL; GODLOVE, 2002).

Embora exista o conhecimento, mesmo que implícito, das relações do aumento do consumo de energia em decorrência dos vazamentos nos SDAs,

'Pontifícia Universidade Católica de Goiás - Goiânia (GO), Brasil.

¿Universidade de Brasilia - Brasília (DF), Brasil.

*Autor correspondente: aksoares@gmail.com

Conflitos de interesse: os autores declaram não haver conflito de interesses.

Financiamento: Conselho Nacional de Desenvolvimento Científico e Tecnológico (CNPq).

Recebido: 23/08/2019 - Aceito: 14/04/202O - Reg. ABES: 20190255 
uma análise direta da questão é aparentemente recente e escassa. As pesquisas relacionadas com a operação de SDAs, tradicionalmente, têm focado a quantificação, a detecção e a redução de vazamentos (ALMANDOZ et al., 2005; ALVISI; FRANCHINI, 2009; NICOLINI; GIACOMELLO; DEB, 2011; CAMPISANO; MODICA; VETRANO, 2012; CREACO; PEZZINGA, 2015), a eficiência hidráulica e outras buscam o uso otimizado de energia nos sistemas (CARRIJO, 2004; BROAD; MAIER; DANDY, 2010; WU et al., 2012; KUREK; OSTFELD, 2014; MALA-JETMAROVA; BARTON; BAGIROV, 2015; ODAN; REIS; KAPELAN, 2015). Outras pesquisas, como a de Pino et al. (2017), buscam, por meio de algoritmos evolucionários multiobjetivo, obter soluções na concepção de SDAs que promovam maior confiabilidade e eficiência hidráulica.

Neste trabalho é apresentado um modelo multiobjetivo para realizar a otimização da operação de SDA com a finalidade de obter regras operacionais adequadas para o funcionamento das bombas existentes no sistema. Tais regras devem promover a eficiência hidroenergética, compreendida como o sinergismo entre as eficiências hidráulica e energética. Como se trata de um problema multiobjetivo, são encontradas diversas soluções, as quais não apresentam dominância umas sobre as outras, dando origem ao que se denomina frente Pareto. Com vistas às análises da aplicação do modelo proposto, empregou-se o SDA hipotético denominado Anytown (WALSKI et al., 1987), o qual é difundido na literatura e foi utilizado na "Batalha dos Modelos de Rede", evento que consistiu em uma série de sessões realizadas em uma conferência na cidade de Nova Iorque, no ano de 1985. Dentre as soluções apresentadas na conferência, selecionou-se aquela desenvolvida por Gessler (1985).

\section{METODOLOGIA}

As diretrizes metodológicas para definir a operação ótima de um SDA seguem cinco etapas distintas, distribuídas de acordo com as duas fases:

- Fase 1: Geração do conjunto de soluções ótimas:

1. Escolha dos objetivos e definição das funções objetivo;

2. Definição dos algoritmos evolucionários multiobjetivo e dos parâmetros e operadores genéticos;

3. Geração das soluções ótimas via algoritmo evolucionário multiobjetivo.

- Fase 2: Escolha da melhor solução:

5. Avaliação das soluções e das relações de compromisso entre as funções objetivo;

6. Ordenação das soluções.

A Fase 1 é realizada por meio de um modelo computacional que integra o simulador hidráulico e os algoritmos genéticos; sua aplicação resulta em um conjunto de soluções não dominadas, denominado frente Pareto. Uma vez que há mais de uma solução, é necessário avaliar cada uma delas, bem como hierarquizá-las a partir de critérios estabelecidos pelo operador do SDA (Decisor); para isso, foram utilizadas outras ferramentas computacionais separadas no modelo computacional na Fase 2.

\section{Definição dos objetivos}

A eficiência hidroenergética de uma rede pode ser obtida mediante regras operacionais que priorizem simultaneamente a redução do volume de vazamentos e a redução do custo de energia elétrica consumida pelas bombas durante o período de operação do sistema, sendo que não deve haver priorização de um desses objetivos em detrimento do outro. Conforme demonstrado por Colombo e Karney (2005), os vazamentos são responsáveis pela perda de eficiência hidráulica, bem como por aumentar o consumo de energia elétrica, por incluir uma demanda extra de pressão e vazão, contribuindo para a perda de eficiência energética.

Colombo e Karney (2005) focaram seus estudos sobre um sistema com abastecimento direto, ou seja, o ramal predial está diretamente ligado à rede de distribuição de água, o que é comum nos SDAs do hemisfério norte. No Brasil, a utilização de reservatórios domiciliares é dominante e, portanto, o abastecimento de água às residências é classificado como indireto. Recentemente, Giustolisi, Laucelli e Berardi (2013) concluíram que, para SDAs com reservatórios domiciliares e com reservatórios distribuídos internamente no sistema, os objetivos de redução de perdas por vazamentos e de minimização dos custos de energia elétrica no bombeamento são conflitantes. Desse modo, é fundamental que ambos os objetivos sejam analisados simultaneamente para avaliação da eficiência hidroenergética.

Quando considerados somente os objetivos de redução de perdas e de custo de energia elétrica, pode ocorrer perda significativa da confiabilidade do sistema em atender às demandas de pressões e vazões nos pontos de consumo de água; logo, é necessário incluir um terceiro objetivo que busque maximizar a confiabilidade da rede, cuja finalidade é garantir que os usuários não sejam prejudicados por regras operacionais que comprometam o abastecimento

Assim, os objetivos escolhidos foram:

- minimização do volume de perdas por vazamento na rede de distribuição de água;

- minimização do custo de energia elétrica durante a operação do sistema (bombeamento);

- maximização da confiabilidade em atender às demandas de vazão e pressão dos usuários.

Foram definidas funções objetivo capazes de quantificar, ou seja, medir cada objetivo, e assim realizar comparações entre diferentes soluções, permitindo selecionar somente as soluções que garantam a eficiência hidroenergética do sistema.

Por se tratar de um problema multiobjetivo, é possível a existência de um conjunto de soluções, em que todas são eficientes e não há dominância de uma sobre a outra, ou seja, para os objetivos avaliados, todas as soluções selecionadas têm desempenho equivalente, assim denominadas soluções não dominadas, conhecidas também como frente de Pareto. Quando uma solução é não dominada, não existe outra solução admissível que melhore, simultaneamente, todas as funções objetivo, isto é, a melhoria em uma função objetivo só pode ser alcançada aceitando piorar o valor de pelo menos uma das outras funções objetivo.

Conforme Pudar e Liggett (1992), Rossman (2000) e Colombo e Karney (2002; 2005), os vazamentos podem ser representados pela equação de orifícios com uma seção constante. No simulador hidráulico EPANET (ROSSMAN, 2000), os parâmetros da equação de orifícios são agrupados em um único coeficiente, denominado coeficiente emissor $(C E)$, enquanto a relação entre a área efetiva do orifício e a pressão interna atuante na tubulação pode ser representada por um expoente de vazamento $(\alpha)$. Para representar os vazamentos em cada nó $n$ da rede ao longo do tempo $\left(q_{n t}\right)$, adotou-se a equação do EPANET (Equação 1),

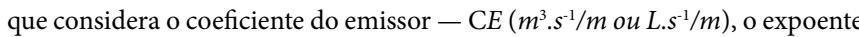
de vazamento $-\alpha$ (adimensional), bem como a carga de pressão $-H(\mathrm{~m})$ : 
$\mathrm{q}_{n t}=C E \cdot H^{\alpha}$

Para verificar a aplicação da Equação 1, bem como definir os valores do coeficiente emissor ( $C E)$ e do expoente de vazamento, foram realizadas simulações do SDA em diferentes cenários. Previamente, optou-se por fixar o expoente em 0,5, conforme sugerido por Rossman (2000). Após simulações e análise, optou-se por adotar $C E$ de 0,204 , pois esse valor resultou em vazamentos na rede de distribuição da ordem de $5 \%$ do volume total de água.

A função objetivo FO1 (Equação 2) tem a finalidade de promover a eficiência hidráulica da rede, buscando o menor índice de vazamentos, o qual é quantificado pela soma das perdas por vazamento $\left(q_{\mathrm{nt}}\right)$ em cada um dos nós (nn) durante as 24 horas da simulação.

$F 01=\min [f 1], f 1=\sum_{t=1}^{24} \sum_{n=1}^{n n} \mathrm{q}_{n t}$

O custo de energia elétrica depende da potência consumida por cada bomba e do preço da tarifa. No Brasil, em razão de a matriz de energia ser predominante hidroelétrica, foi estabelecida pela Agência Nacional de Energia Elétrica (Aneel) uma estrutura tarifária complexa que faz distinção do tipo de consumidor, do período do ano e da hora de maior consumo no dia (tarifa de horário de pico e tarifa fora do horário de pico). O tipo de consumo considerado para os SDAs é de serviço público, o qual segue uma estrutura de tarifa horossazonal. Nesse sistema, o custo da energia é calculado em função da demanda de potência contratada previamente e do consumo ao longo do dia. Considerando-se a estrutura tarifária horossazonal, o custo de energia elétrica para operação de 24 horas do SDA é determinado pela Equação 3 .

$C F E_{t}=\sum_{b=1}^{n_{b}} \frac{Q_{b} H_{b} \gamma}{\eta_{b}} T C_{t}+\frac{T D \cdot D c}{30}+T U_{t}(D m-D c)$

Sendo:

$C F E=$ o custo total do fornecimento de energia elétrica (Int\$ - dólar internacional); $\mathrm{n}_{\mathrm{b}}=\mathrm{o}$ número de bombas;

$Q_{\mathrm{b}}=$ a vazão bombeada $\left(\mathrm{m}^{3} / \mathrm{s}\right)$;

$H_{\mathrm{b}}=\mathrm{a}$ altura manométrica de bombeamento $(\mathrm{m})$;

$\eta_{\mathrm{b}}=\mathrm{o}$ rendimento do conjunto motor-bomba;

$\gamma=$ o peso específico da água $\left(\mathrm{kN} / \mathrm{m}^{3}\right)$, o qual permite determinar a potência consumida;

$n_{\mathrm{b}}=\mathrm{o}$ número de bombas funcionando na estação elevatória;

$T C=$ a tarifa unitária de consumo de energia no período de uma hora $(\mathrm{Int} \$ / \mathrm{kW})$;

$T D=$ a tarifa de demanda de potência contratada (Int $\$ / \mathrm{kW})$;

$D c=$ a demanda máxima de potência contratada $(\mathrm{kW})$;

$T U=$ a tarifa de ultrapassagem da demanda de potência contratada (Int $\$ / \mathrm{kW})$; $D m=$ a demanda máxima de potência consumida $(\mathrm{kW})$.

A função objetivo FO2 (Equação 4) visa maximizar a eficiência energética mediante a minimização do custo total de fornecimento de energia elétrica (CFE), reduzindo simultaneamente a quantidade de energia elétrica consumida e a demanda de potência utilizada pelo sistema.

$F 02=\min [f 2], f 2=\sum_{t=1}^{24} C F E_{t}$
Para realizar as simulações do custo de energia elétrica, adotou-se uma estrutura tarifária horossazonal verde (ANEEL, 2010), em que os custos da tarifa são apresentados, em dólar internacional (Int\$), na Tabela 1. Ressalta-se que cada companhia de energia elétrica define os valores de suas tarifas e, portanto, para aplicação em sistemas de abastecimento de água existentes, deve-se consultar os valores dessas tarifas.

A vazão a ser entregue em cada nó, com pressão adequada, é o principal fator na determinação da confiabilidade de uma rede de distribuição de água, uma vez que a vazão e a pressão são fundamentais para garantir o perfeito atendimento dos usuários. Todini (2000) apresenta uma medida heurística que busca determinar a confiabilidade do SDA por meio do conceito de resiliência, variando de 0 a 1 . Esse conceito foi elencado para representar a confiabilidade da rede, pois, conforme Formiga (2005), a base central desse conceito é a capacidade do sistema em atender às demandas dos consumidores. Além disso, Cheung (2004) aponta que a formulação da confiabilidade por meio da resiliência tem grande facilidade computacional para o seu cálculo. A resiliência, neste trabalho, é calculada pela Equação 5:

$R=\sum_{1}^{24} \frac{\sum_{i=1}^{n n} Q_{i} \times\left(H_{i}-H_{d e s}\right)}{\sum_{i=1}^{n t} Q_{r} \times H_{r}-\sum_{i=1}^{n n} Q_{i} \times H_{d e s}}$

Sendo:

$R=\mathrm{o}$ índice de confiabilidade acumulada (resiliência) durante as 24 horas de operação do sistema (logo, o valor varia de 0 a 24 );

$n n=$ o número total de nós;

$Q_{\mathrm{i}}=$ a demanda abastecida no nó $i(\mathrm{~L} / \mathrm{s})$;

$H_{\mathrm{i}}=$ a carga de pressão fornecida ao nó $i(\mathrm{~m})$;

$H_{\text {des }}=$ a carga de pressão desejada para que a demanda seja totalmente abastecida $(\mathrm{m})$;

$n_{\mathrm{t}}=\mathrm{o}$ número de reservatórios;

$Q_{\mathrm{r}}=$ a vazão abastecida pelo reservatório $t(\mathrm{~L} / \mathrm{s})$;

$H_{\mathrm{r}}=$ a carga de pressão fornecida pelo reservatório $t(\mathrm{~m})$.

A função objetivo FO3 (Equação 6) busca garantir o atendimento das demandas de acordo com as pressões requeridas nos nós de consumo por meio da maximização da resiliência $(R)$.

$F 03=\max [f 3], f 3=R$

\section{Algoritmos evolucionários multiobjetivo}

A evolução dos algoritmos genéticos veio com os algoritmos evolucionários multiobjetivo (AEMO), também chamados de algoritmos genéticos multiobjetivo. Essa técnica permite a otimização de várias funções objetivo, buscando

Tabela 1 - Valores de tarifa horossazonal verde.

\begin{tabular}{l|c|c|}
\multirow{2}{*}{ Tarifa horossazonal verde } \\
\cline { 2 - 3 } Consumo & $\begin{array}{c}\text { Fora de pico - TC } \\
(\text { Int\$/kWh) }\end{array}$ & Pico - TC (Int\$/kWh) \\
\cline { 2 - 3 } & 0,09687 & 0,47266 \\
\hline Demanda - TD & \multicolumn{2}{|c|}{$3,694(\mathrm{Int} \$ / \mathrm{kWh})$} \\
\hline Demanda de ultrapassagem - TU & \multicolumn{2}{|c|}{$7,388(\mathrm{Int} \$ / \mathrm{kWh})$} \\
\hline
\end{tabular}

Fonte: elaboração própria. 
o melhor desempenho do sistema por meio da geração das soluções não dominadas, chamada de frente Pareto.

Com o objetivo de obter as melhores soluções, ou seja, a frente Pareto, para o problema de eficiência hidroenergética representado neste trabalho pelas três funções objetivo descritas, foram utilizados os algoritmos evolucionários multiobjetivo elitistas SPEA (Strength Pareto Evolutionary Algorithm) e NSGAII (Non-dominated Sorting Genetic Algorithm), visto que em outros trabalhos ficou demonstrada a superioridade de suas as soluções apresentadas (CARRIJO, 2004; CHEUNG, 2004; FORMIGA, 2005). Os algoritmos foram avaliados neste trabalho e as soluções fornecidas por cada um foram transformadas em regras operacionais.

O SPEA é um algoritmo evolucionário multiobjetivo desenvolvido por Zitzler e Thiele (1998). Esse algoritmo, além de utilizar o conceito de frente Pareto, usa técnicas elitistas para seleção das melhores soluções. O elitismo é considerado por meio de uma população externa em que são guardadas as soluções não dominadas encontradas ao longo do processo. O NSGA II foi elaborado por Deb et al. (2000) e emprega conceitos do método NSGA (SRINIVAS; DEB, 1994), mas inclui o conceito de elitismo, preservando as melhores soluções.

\section{Modelo para simulação hidráulica}

Foi elaborado um simulador hidráulico, baseado na versão Toolkit do software EPANET e codificado na linguagem computacional C++. A Toolkit é uma biblioteca de vínculo dinâmico (DLL) que contém as funções que permitem o desenvolvimento de tarefas específicas contidas no EPANET. O modelo de simulação visa realizar as simulações da operação de uma rede de abastecimento de água durante 24 horas, considerando intervalos de tempo de uma hora para calcular os valores de cada função objetivo. A Figura 1 mostra as etapas do modelo hidráulico.

O início do simulador hidráulico é realizado a partir de um arquivo de entrada (.inp), o qual fornece as condições topológicas da rede de abastecimento e os parâmetros iniciais da rede, como a demanda média em cada nó, o nível inicial dos reservatórios, o comprimento e o diâmetro dos tubos, a cota topográfica dos nós, as curvas características das bombas.

A demanda nos nós $\left(Q_{n}\right)$, que corresponde à água fornecida aos consumidores, é obtida a partir da demanda média multiplicada por um fator temporal (FT), o qual representa, em cada hora, a variação temporal do consumo de água. Além da demanda, há em cada nó uma vazão que corresponde às perdas por vazamento $\left(q_{\mathrm{nt}}\right)$, calculada conforme o modelo de vazamento explicitado na Equação 1. A vazão total distribuída ao longo do dia $\left(Q_{\mathrm{d}}\right)$ corresponde ao somatório da demanda em cada nó $\left(Q_{\mathrm{n}}\right)$ mais o somatório dos vazamentos nos nós $\left(q_{\mathrm{nt}}\right)$, conforme a seguinte relação (Equação 7):

$Q_{d}=\sum_{t=1}^{24} \sum_{1}^{n} Q_{n} * F T_{t}+\sum_{t=1}^{24} \sum_{n=1}^{n n} q_{n t}$

Para que o modelo seja capaz de calcular a vazão total considerando a demanda, o fator temporal e os vazamentos na rede, bem como realizar a alteração dos níveis dos reservatórios, foi desenvolvido um loop de avaliação que calcula o somatório da diferença entre as pressões na iteração anterior e as pressões na iteração atual para cada nó. O resultado da diferença é o desvio total, e a simulação sai desse loop somente quando o desvio total for menor que $0,001 \mathrm{~m}$. O simulador hidráulico, após o loop de avaliação, realiza o balanço hídrico do SDA e calcula o valor de cada função objetivo para cada hora. Após as 24 horas, é realizado o somatório total de todas as funções objetivo, as quais são utilizadas para realizar a otimização.

\section{Modelo para otimização}

O modelo de otimização multiobjetivo proposto consiste na integração dos algoritmos evolucionários multiobjetivo (SPEA e NSGAII) e do simulador hidráulico, ambos codificados na linguagem $\mathrm{C}++$, além do modelo de classificação das soluções (PROMETHEE II). A integração dos modelos é ilustrada na Figura 2.

Para obter regras operacionais que priorizem a eficiência hidroenergética, foi considerado como variável de decisão o estado (status), ligado ou desligado, das bombas em cada hora, durante o período de planejamento de 24 horas. Cada vetor solução para o problema é representado por uma codificação binária (0 ou 1). O zero (0) representa a bomba que está desligada em um intervalo de tempo, enquanto o um (1) representa a bomba que está ligada. Dessa forma, o número de indivíduos que um vetor solução contém corresponde ao número de bombas existentes na rede multiplicado pelo número de horas no período de planejamento (24 horas).

\section{Ordenamento das soluções}

Conforme indicado na Figura 2, o modelo computacional, por meio dos algoritmos evolucionários multiobjetivo, apresenta um conjunto de soluções não

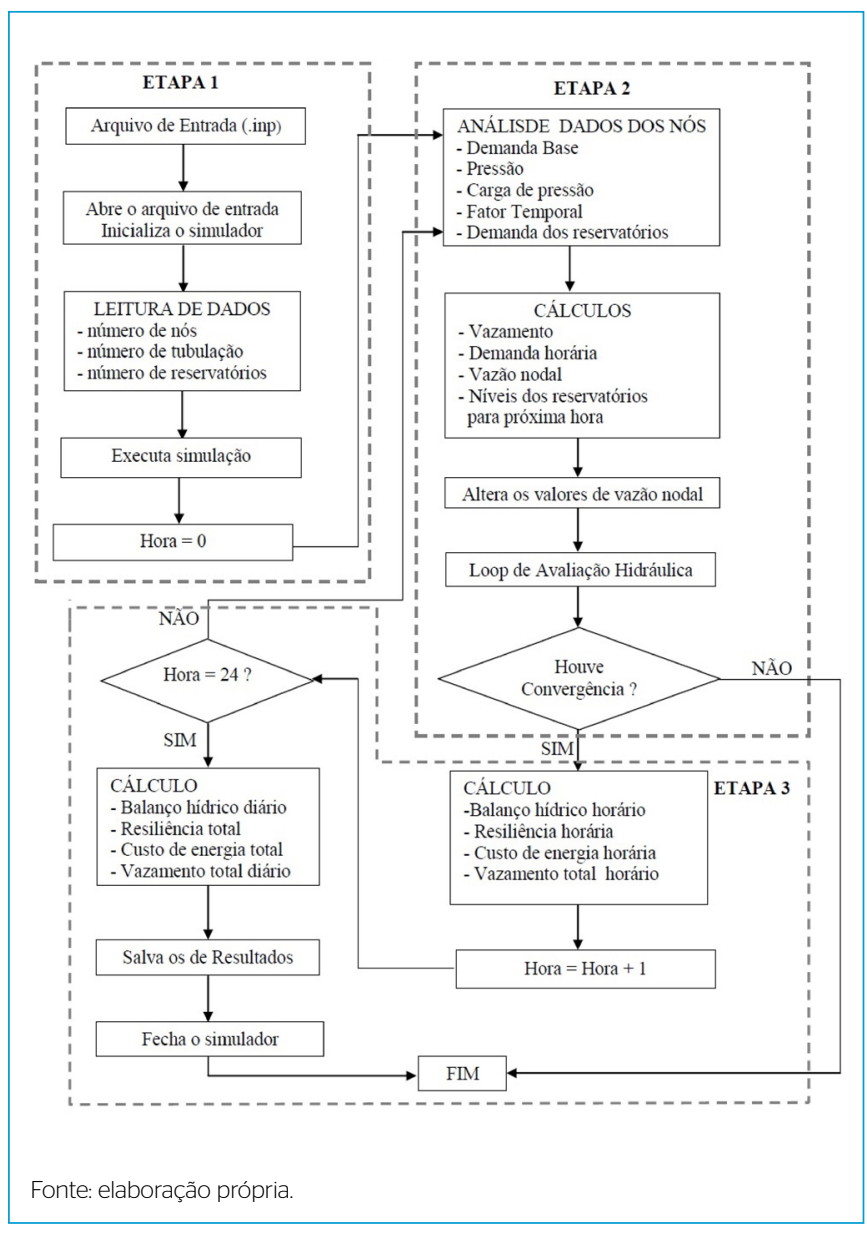

Figura 1 - Etapas da simulação hidráulica. 
dominadas. Todavia, não é possível que todas as soluções sejam executadas ao mesmo tempo. Assim, é necessário que o responsável pela operação do sistema opte por uma única solução. Para auxiliar o decisor na escolha da melhor solução a ser empregada, existem metodologias específicas, como, por exemplo, o método PROMETHEE (Preference Ranking Organization Methods for Enrichment Evaluations), proposto por Brans, Vincke e Mareschal (1986). Com base em critérios preestabelecidos pelo decisor, esse método estabelece uma estrutura de hierarquização das soluções a partir dos critérios mais significativos estabelecidos pelo decisor.

Na Fase 2 deste trabalho, para realizar a ordenação das soluções, adotou-se o método PROMETHEE II. O método foi implementado a partir de uma rotina computacional escrita em Visual Basic 6.0, desenvolvida por Jannuzzi (2010), denominada como Programa para Apoio à Tomada de Decisão Baseada em Indicadores (PRADIN). Essa rotina não é vinculada ao modelo computacional, sendo que ela é realizada após a definição das soluções.

\section{Estudo de caso}

Com vistas às análises do modelo proposto, empregou-se a rede hipotética Anytown utilizada na "Batalha dos Modelos de Rede", evento que consistiu em uma série de sessões realizadas em uma conferência na cidade de Nova Iorque, no ano de 1985. Dentre as soluções apresentadas nessa conferência por Walski et al. (1987), selecionou-se aquela desenvolvida por Gessler (1985). A justificativa para a escolha dessa rede foi em decorrência de sua utilização em diversos trabalhos de pesquisa (BRILL; LIEBMAN; LEE, 1985; GESSLER, 1985; LANSEY; MAYS, 1985; MORGAN; GOULTER, 1985; ORMSBEE, 1985; COLOMBO; KARNEY, 2005) que objetivaram reduzir os custos de implantação e operação da rede. A solução desenvolvida por Gessler (1985) foi selecionada por ser utilizada em

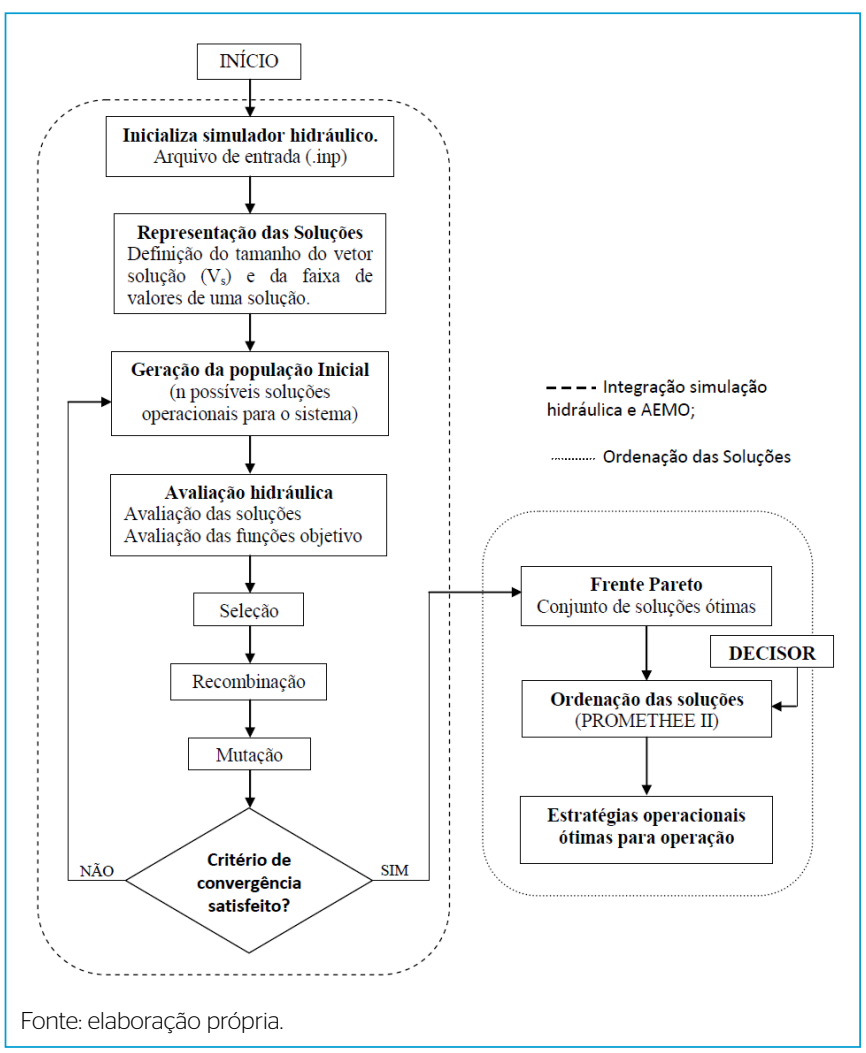

Figura 2 - Fluxograma do modelo computacional. outros trabalhos, como os de Colombo e Karney $(2002 ; 2005)$. A topologia da rede Anytown é apresentada na Figura 3. A rede é composta por 40 trechos, tubulações de ferro fundido, 3 reservatórios (tanks), 19 nós com demanda e 3 bombas ligadas em paralelo, as quais foram inseridas neste trabalho, considerando a potência total apresentada por Walski et al. (1987). Adaptou-se a rede também para um cenário com $5 \%$ de perdas por vazamento.

Conforme observa-se na Figura 3, a rede foi adaptada para a realidade dos SDAs brasileiros, nos quais a distribuição de água é realizada, geralmente, por gravidade a partir de um reservatório a montante. Logo, adicionou-se um reservatório a montante da rede de distribuição (tank 10), o qual fornece toda a água à rede de distribuição. A definição do volume desse reservatório foi determinada com base na curva de consumo apresentada por Walski et al. (1987). A capacidade total de reservação, considerando-se o reservatório de montante e os de posição intermediária, totaliza $20 \%$ do volume total distribuído diariamente.

\section{RESULTADOS}

Visando verificar a eficiência do modelo de otimização proposto, empregou-se, com adaptações, o SDA Anytown.

Para gerar o conjunto de soluções da frente Pareto, os parâmetros e operadores genéticos adotados para ambos os algoritmos (SPEA e NSGAII) foram:

- $\quad$ população inicial $=100$;

- $\quad$ número de gerações = 1.000;

- taxa de mutação = 0,1;

- $\quad$ taxa de recombinação $=0,9$.

Tais parâmetros foram definidos via utilização da métrica Set Coverage. Foram realizadas sete simulações para cada algoritmo.

Para o algoritmo NSGAII, a frente Pareto produzida gerou 23 soluções, as quais representam as melhores estratégias operacionais obtidas por esse algoritmo para a rede de distribuição de água nas simulações realizadas. Os valores das funções objetivo são apresentados na Tabela 2. A Figura 4 apresenta as soluções encontradas no espaço de busca.

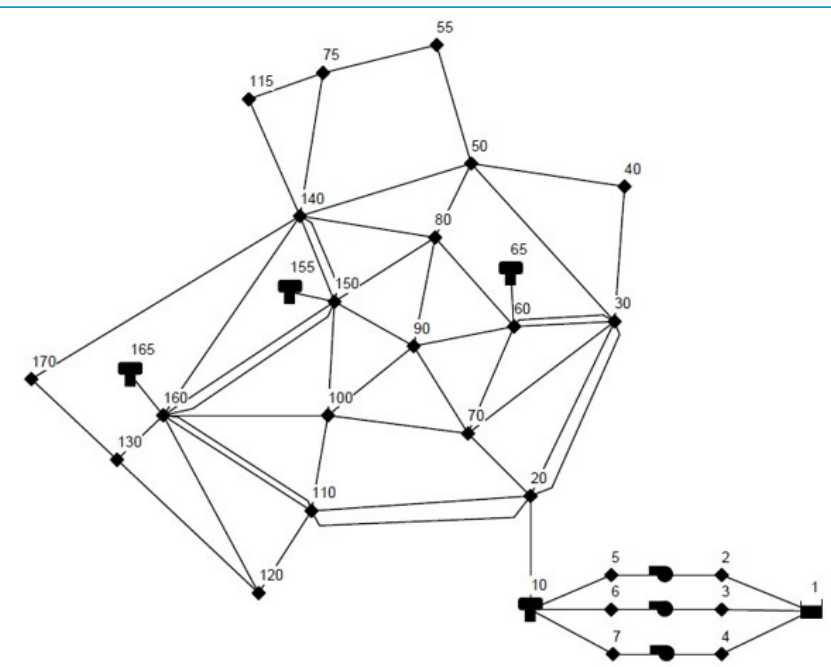

Fonte: elaboração própria.

Figura 3 - Sistema de distribuição de água Anytown, com adaptações. 
Tabela2 - Conjunto de soluções obtidas com Non-dominated Sorting Genetic Algorithm.

\begin{tabular}{|c|c|c|c|}
\hline Solução & $\mathrm{FO} 1\left(\mathrm{~m}^{3} / \mathrm{dia}\right)$ & FO2 (Int\$) & FO3 (-) \\
\hline 1 & $64.815,0$ & $1.301,24$ & 16,8268 \\
\hline 2 & $64.309,8$ & $1.354,47$ & 16,343 \\
\hline 3 & $64.295,4$ & 1.393,11 & 16,2754 \\
\hline 4 & $64.811,7$ & $1.356,22$ & 16,4986 \\
\hline 5 & $64.648,7$ & 2.071,79 & 17,0326 \\
\hline 6 & $65.556,0$ & $2.006,23$ & 17,4929 \\
\hline 7 & $65.452,4$ & $2.195,32$ & 17,7301 \\
\hline 8 & $64.640,9$ & $2.503,45$ & 17,0401 \\
\hline 9 & $65.079,6$ & 793,92 & 16,5761 \\
\hline 10 & $67.116,2$ & $1.201,29$ & 16,6267 \\
\hline 11 & $65.548,1$ & $1.512,08$ & 17,3583 \\
\hline 12 & $64.367,8$ & $1.446,13$ & 16,8253 \\
\hline 13 & $65.500,4$ & $1.950,89$ & 17,1835 \\
\hline 14 & $65.165,0$ & $1.856,7$ & 17,0727 \\
\hline 15 & $67.116,2$ & $1.201,29$ & 16,6267 \\
\hline 16 & $65.548,1$ & $1.512,08$ & 17,3583 \\
\hline 17 & $64.367,8$ & $1.446,13$ & 16,8253 \\
\hline 18 & $65.500,4$ & $1.950,89$ & 17,1835 \\
\hline 19 & $64.808,3$ & $2.038,41$ & 17,4523 \\
\hline 20 & $65.059,6$ & $1.637,29$ & 16,9287 \\
\hline 21 & $64.243,2$ & $1.594,88$ & 15,4525 \\
\hline 22 & $64.820,7$ & 942,32 & 16,5347 \\
\hline 23 & $64.228,7$ & $1.797,55$ & 15,9967 \\
\hline
\end{tabular}

Fonte: elaboração própria.
O algoritmo SPEA gerou uma frente Pareto com 29 soluções, as quais são apresentadas na Tabela 3 e na Figura 5. Verifica-se que, em algumas soluções, a redução do custo de energia elétrica (isto é, diminuição do bombeamento na rede) não implicou diminuição das perdas por vazamentos, o que demonstra que tais objetivos podem ser conflitantes para SDAs com vários reservatórios, conforme afirmado inicialmente por Giustolisi, Laucelli e Berardi (2013).

\section{Ordenamento das soluções e geração de regras operacionais}

Uma regra operacional ótima consiste na implementação de apenas uma solução. Visando selecionar apenas uma solução contida na frente Pareto, foi realizada a ordenação das soluções por meio do método PROMETHEE II, com a utilização do aplicativo PRADIN 3.0. A ordenação das soluções é realizada a partir do índice de preferência global, o qual permite determinar fluxos de importância positivo (que demonstra a força da solução A sobre a solução B) e negativo (demonstra a fraqueza da solução $\mathrm{A}$ em relação à solução $\mathrm{B})$, resultando no índice multicritério (IMC) do aplicativo. Quanto maior o valor do índice, mais próxima a solução está da preferência do decisor.

Foram atribuídos pesos iguais para cada função objetivo, visando gerar as regras operacionais para cada uma das frentes Pareto. Ressalta-se que pesos diferentes podem ser estabelecidos, permitindo que os tomadores de decisão estabeleçam seus próprios critérios. Entretanto, o estudo específico de adoção dos pesos das funções objetivo não está no escopo deste trabalho.

A partir da ordenação das soluções geradas pelo NSGAII, verifica-se, na Figura 6 e por meio dos valores do IMC, que as melhores soluções são as de
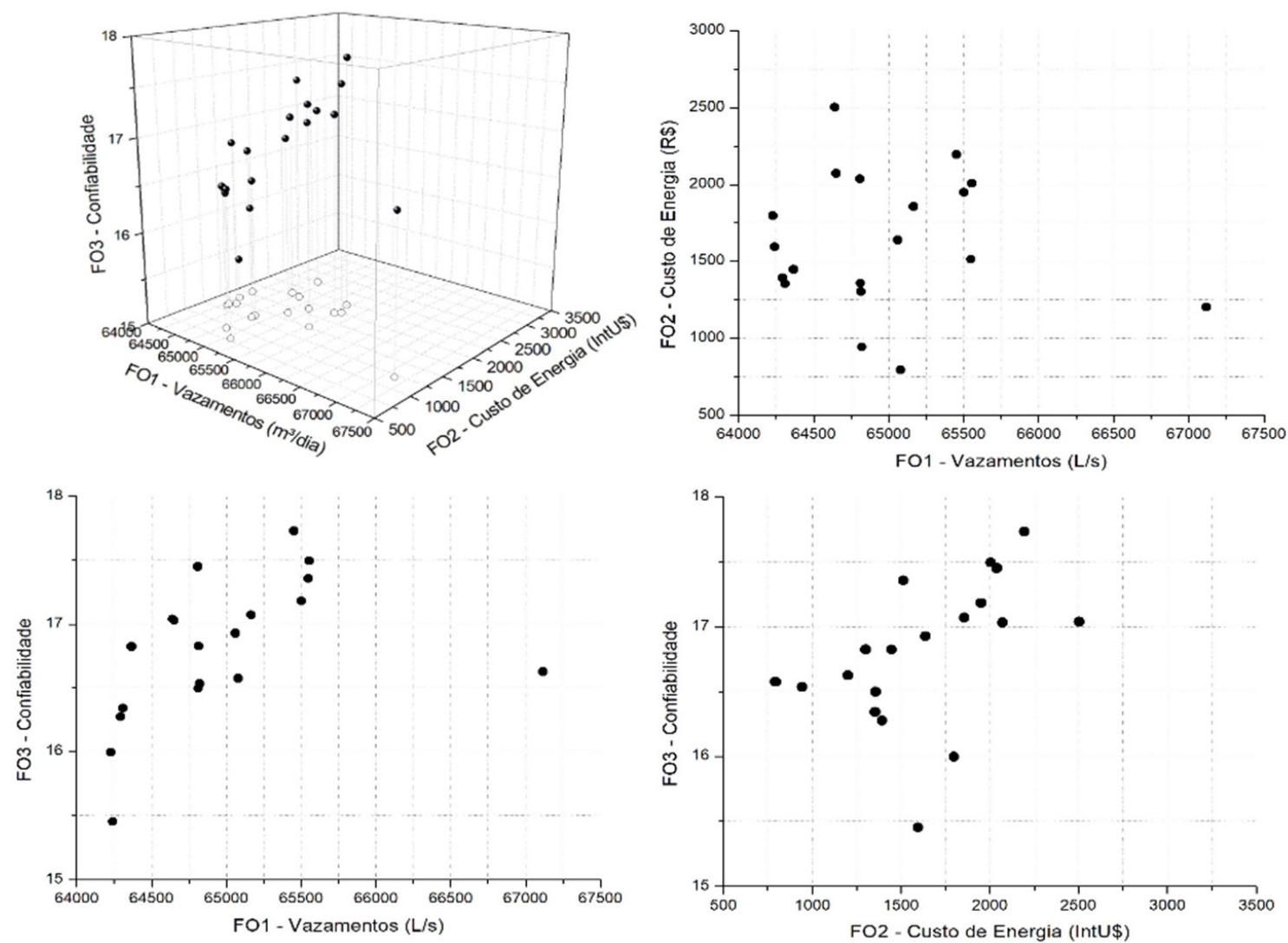

Fonte: elaboração própria.

Figura 4 - Representação gráfica da frente Pareto: Non-dominated Sorting Genetic Algorithm. 
Tabela3-Conjunto de soluções obtidas com o Strength Pareto Evolutionary Algorithm.

\begin{tabular}{c|c|c|c}
\hline Solução & FO1 ( $\left.\mathbf{m}^{3} / \mathrm{dia}\right)$ & FO2 (U\$) & FO3 (-) \\
\hline 1 & $64.874,3$ & $1.804,42$ & 16,8174 \\
\hline 2 & $65.324,7$ & $1.814,70$ & 17,6118 \\
\hline 3 & $64.719,3$ & $1.651,75$ & 16,6439 \\
\hline 4 & $65.321,8$ & $1.152,44$ & 16,2353 \\
\hline 5 & $64.858,1$ & $1.618,61$ & 16,8105 \\
\hline 6 & $65.110,9$ & $1.824,18$ & 16,9162 \\
\hline 7 & $64.901,7$ & $1.361,92$ & 16,3698 \\
\hline 8 & $65.847,8$ & $1.673,94$ & 17,169 \\
\hline 9 & $65.787,4$ & $1.730,82$ & 17,2862 \\
\hline 10 & $66.970,3$ & $1.488,18$ & 16,9893 \\
\hline 11 & $64.501,7$ & $1.680,74$ & 16,4931 \\
\hline 12 & $65.018,4$ & $1.986,31$ & 17,1724 \\
\hline 13 & $66.451,4$ & $1.498,98$ & 17,2863 \\
\hline 14 & $65.150,9$ & $1.789,13$ & 17,3781 \\
\hline 15 & $65.534,6$ & $1.569,94$ & 17,0654 \\
\hline 16 & $64.922,0$ & $2.381,14$ & 17,2224 \\
\hline 17 & $65.037,7$ & $1.558,41$ & 16,4112 \\
\hline 18 & $64.916,9$ & $2.509,46$ & 17,3744 \\
\hline 19 & $65.112,1$ & $1.569,91$ & 16,5393 \\
\hline 20 & $65.463,1$ & $1.488,93$ & 16,5165 \\
\hline 21 & $64.253,7$ & $1.506,08$ & 16,358 \\
\hline 22 & $65.449,6$ & $1.867,70$ & 17,6188 \\
\hline 23 & $65.618,8$ & $1.468,33$ & 16,5807 \\
\hline 24 & $64.881,8$ & $1.422,97$ & 16,1412 \\
\hline 25 & $64.346,3$ & $1.862,47$ & 16,1777 \\
\hline 26 & $65.389,6$ & 788,90 & 16,2192 \\
\hline 27 & $64.915,2$ & $2.190,93$ & 16,9567 \\
\hline 28 & $65.836,4$ & $1.222,13$ & 16,7394 \\
\hline 29 & $66.024,5$ & $1.355,97$ & 16,8362 \\
\hline & & & \\
\hline
\end{tabular}

Fonte: elaboração própria. número 1 e 16, com iguais valores para IMC $(0,2424)$. O pior valor do IMC $(-0,1818)$ foi atribuído para as soluções 6 e 17.

Para as soluções geradas pelo SPEA, observa-se, na Figura 7, que a solução 5 tem maior $\operatorname{IMC}(0,2143)$, sendo que o pior IMC foi atribuído à solução $25(-0,2143)$.

\section{Análise das regras operacionais}

Foram selecionadas as soluções que apresentaram maior IMC para o NSGAII (solução 16) e SPEA (solução 5) para serem transformadas em regras operacionais. Os valores das funções objetivo de cada regra operacional são apresentados na Tabela 4. Os estados das bombas para cada regra operacional são apresentados na Figura 8 (NSGAII e SPEA).

Visando comparar os resultados obtidos, foi realizada uma simulação hidráulica na qual todas as bombas foram mantidas ligadas. A grande maioria das companhias de saneamento opera seus sistemas dessa maneira visando garantir maior confiabilidade do sistema, caso ocorra alguma falha no fornecimento de energia elétrica. Assim, essa regra operacional foi denominada como "operação extrema”.

Para verificar o comportamento hidroenergético do SDA, foram analisados, para cada hora, os níveis dos reservatórios, os valores da função resiliência e o custo de energia elétrica. Ainda, para verificar as condições de operação da rede de distribuição de água, foram analisadas as cargas de pressões em cada nó, visando constatar se, em algum momento, ocorrem cargas de pressão abaixo de $15 \mathrm{~m}$, valor mínimo estabelecido na função de confiabilidade. Para isso, selecionou-se a hora do dia mais desfavorável, quando há maior consumo de água e na qual foram observados os menores valores para as pressões.

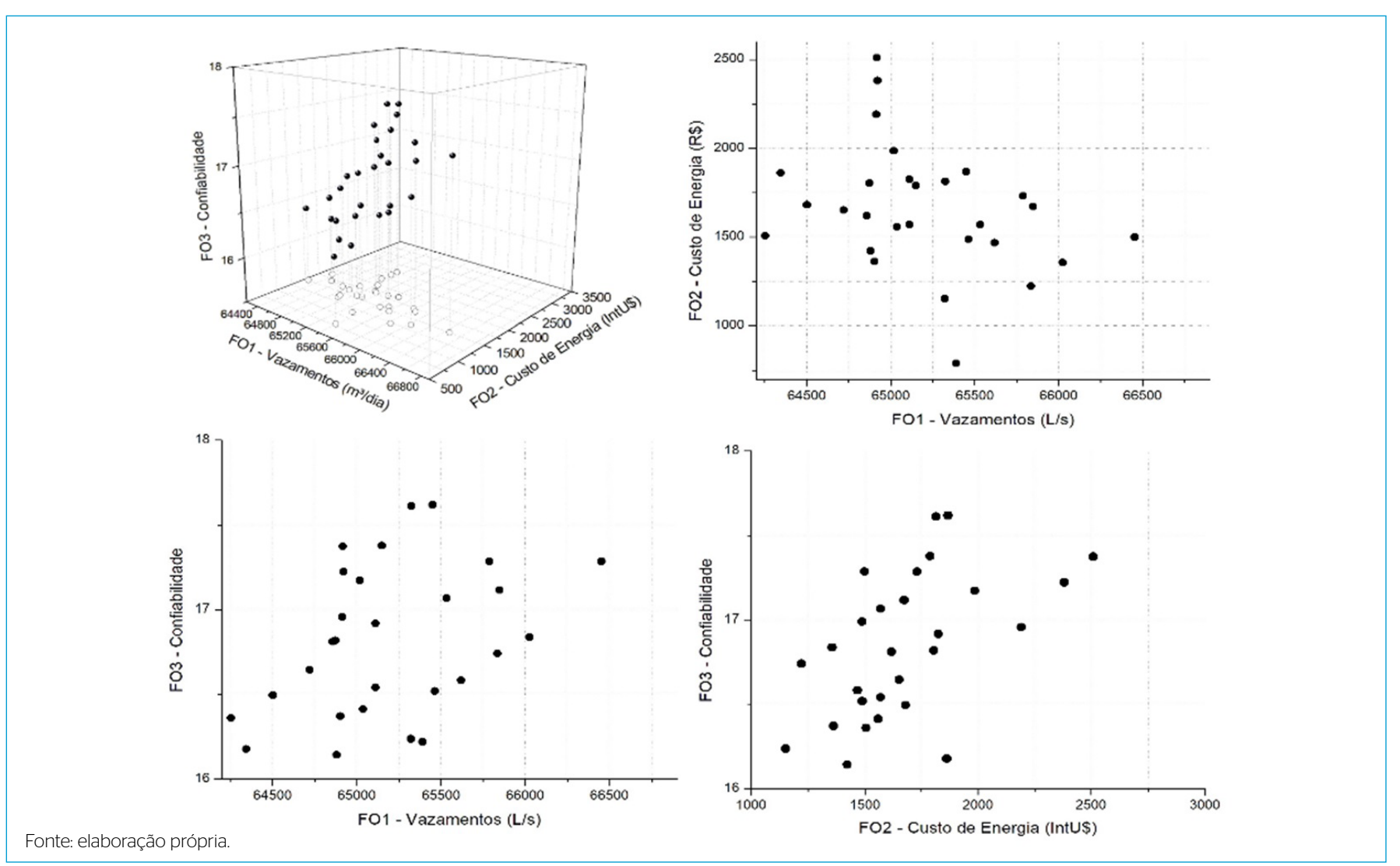

Figura 5 - Representação gráfica da frente Pareto: Strength Pareto Evolutionary Algorithm. 
A Figura 9 mostra o comportamento horário dos reservatórios 65, 155 e 165, a montante da rede de distribuição de água (10). Observa-se que, com uma regra operacional extrema, os reservatórios permanecem, em grande parte do tempo, com os níveis próximos do máximo, enquanto com as regras operacionais ótimas esse nível é mantido próximo ao mínimo em boa parte do dia. Destaca-se que foi definida uma regra para manter um nível mínimo dos reservatórios. No entanto, manter um nível mínimo de água nos reservatórios não significou necessariamente aumento na confiabilidade do sistema, o que ficou demonstrado com a "operação extrema", que, dentre todas as regras operacionais, tem o menor valor para a confiabilidade.
Tabela 4 - Conjunto de regras operacionais.

\begin{tabular}{l|c|c|c|c} 
Regra & AEMO (solução) & FO1 (m³/dia) & FO2 (Int\$) & FO3 \\
$\begin{array}{l}\text { Regra } \\
\text { Operacional }\end{array}$ & NSGAll (16) & $64.367,1$ & $1.446,13$ & 16,82 \\
\cline { 2 - 5 } & SPEA (5) & $64.857,9$ & $1.681,97$ & 16,81 \\
\hline $\begin{array}{l}\text { Operação } \\
\text { Extrema }\end{array}$ & - & $68.627,3$ & $3.063,84$ & 15,20 \\
\hline
\end{tabular}

NSGAll: Non-dominated Sorting Genetic Algorithm; SPEA: Strength Pareto Evolutionary Algorithm

Fonte: elaboração própria.

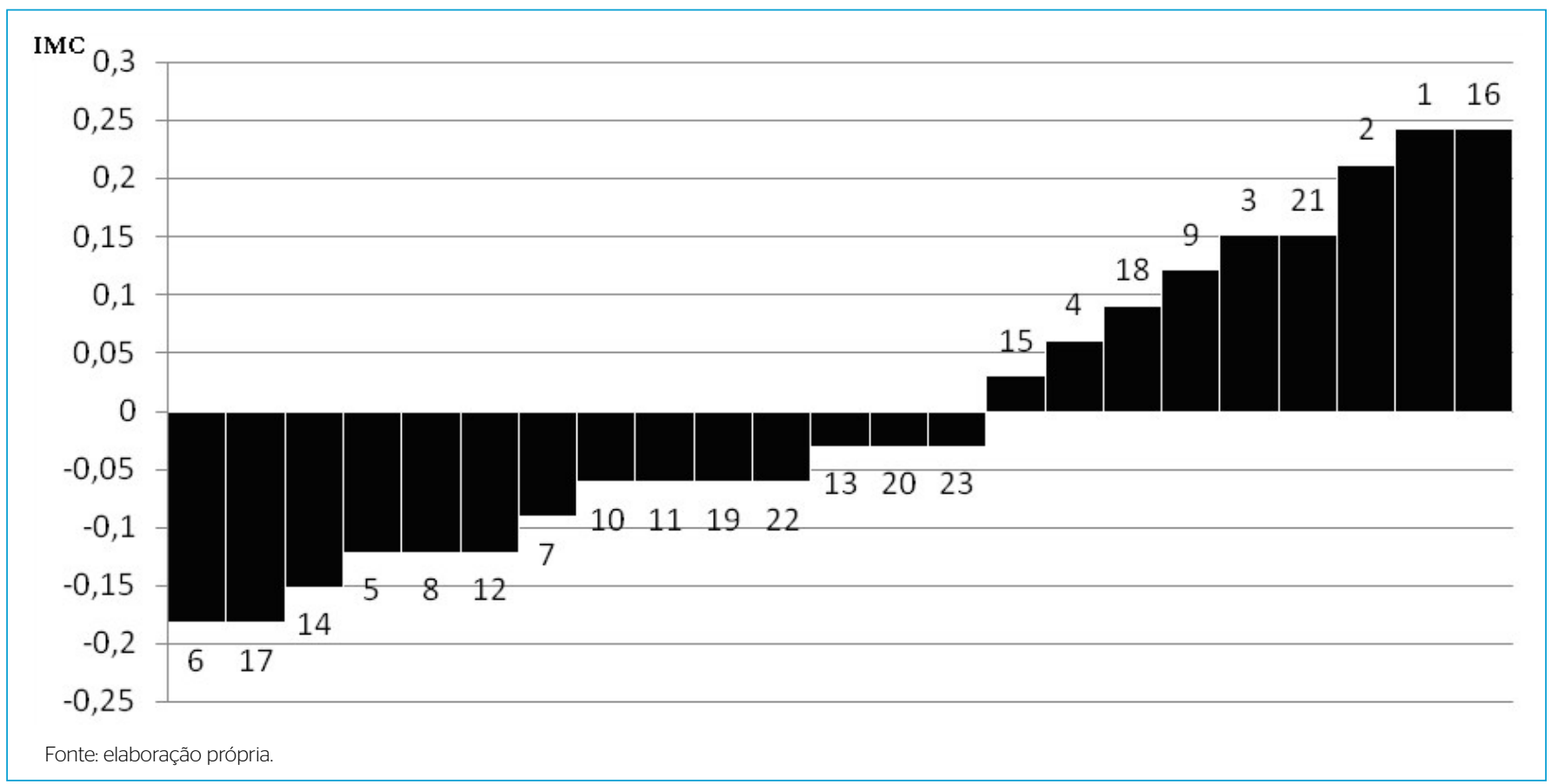

Figura 6 - Ordenação das soluções do Non-dominated Sorting Genetic Algorithm.

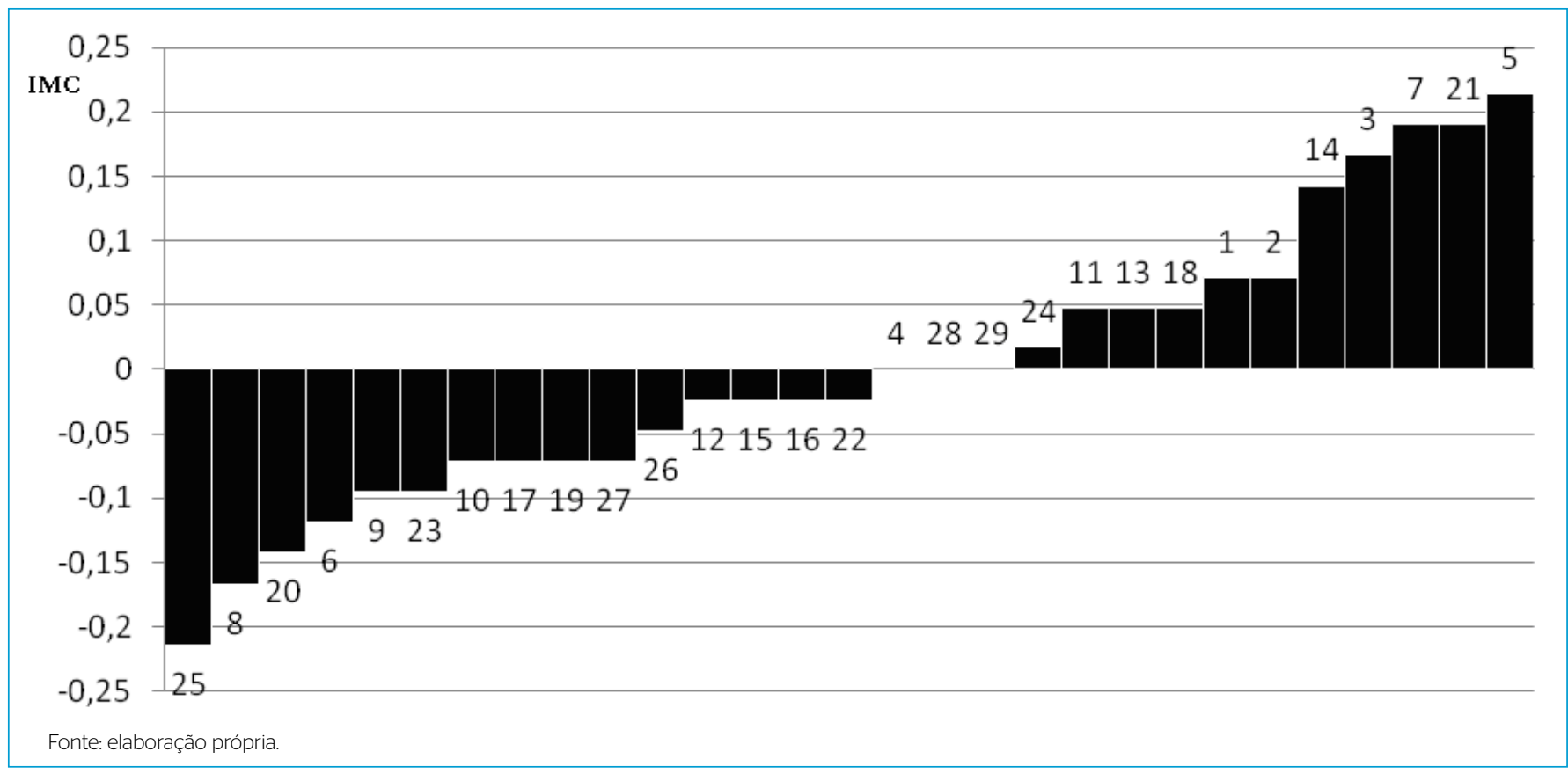

Figura 7 - Ordenação das soluções do Strength Pareto Evolutionary Algorithm. 


\begin{tabular}{|c|c|c|c|c|c|c|}
\hline \multirow{2}{*}{ Hora } & \multicolumn{3}{|c|}{ NSGAII } & \multicolumn{3}{|c|}{ SPEA } \\
\hline & B1 & B2 & B3 & B1 & B2 & $B=$ \\
\hline 0 & 1 & 0 & 0 & 1 & 1 & 0 \\
\hline 1 & 1 & 1 & 0 & 0 & 0 & 0 \\
\hline 2 & 1 & 0 & 0 & 1 & 0 & 0 \\
\hline 3 & 0 & 0 & 0 & 1 & 1 & 0 \\
\hline 4 & 0 & 0 & 0 & 0 & 0 & 0 \\
\hline 5 & 1 & 0 & 0 & 1 & 1 & 1 \\
\hline 6 & 0 & 0 & 0 & 0 & 0 & 0 \\
\hline 7 & 1 & 0 & 0 & 1 & 1 & 1 \\
\hline 8 & 1 & 0 & 0 & 1 & 0 & 0 \\
\hline 9 & 0 & 0 & 0 & 1 & 0 & 0 \\
\hline 10 & 1 & 1 & 1 & 1 & 0 & 0 \\
\hline 11 & 1 & 0 & 0 & 0 & 0 & 0 \\
\hline 12 & 1 & 1 & 1 & 1 & 1 & 0 \\
\hline 13 & 1 & 0 & 0 & 1 & 0 & 0 \\
\hline 14 & 1 & 1 & 0 & 1 & 0 & 0 \\
\hline 15 & 1 & 1 & 0 & 1 & 1 & 0 \\
\hline 16 & 1 & 1 & 0 & 1 & 1 & 0 \\
\hline 17 & 1 & 1 & 0 & 1 & 0 & 0 \\
\hline 18 & 1 & 1 & 0 & 1 & 0 & 0 \\
\hline 19 & 0 & 0 & 0 & 1 & 0 & 0 \\
\hline 20 & 0 & 0 & 0 & 1 & 0 & 0 \\
\hline 21 & 1 & 1 & 0 & 1 & 0 & 0 \\
\hline 22 & 1 & 1 & 0 & 1 & 1 & 0 \\
\hline 23 & 0 & 0 & 0 & 1 & 0 & 0 \\
\hline \multicolumn{7}{|c|}{$\begin{array}{l}\text { Fonte: elaboração própria. } \\
\text { NSGAll: Non-dominated Sorting Genetic Algorithm; SPEA: Strength Pareto } \\
\text { Evolutionary Algorithm. }\end{array}$} \\
\hline
\end{tabular}

Figura 8 - Regras operacionais: estado das bombas.
A Figura 10 apresenta os valores horários para cada um dos objetivos da eficiência hidroenergética. Observa-se que, embora a quantidade de vazamentos na rede seja baixa, as soluções apresentadas pelo modelo apresentam redução significativa na quantidade de vazamentos em cada hora, o que pode ser confirmado ao comparar a curva de vazamentos para uma operação com todas as bombas ligadas com as curvas obtidas pelas soluções NSGAII e SPEA.

No tocante ao custo de energia elétrica, verifica-se que a tarifa no horário de pico (18, 19 e 20 horas) exerce grande influência no custo desse insumo. Assim, as regras operacionais ótimas evitam realizar o bombeamento nesse período. Pelas curvas do custo de energia elétrica apresentadas para cada solução, verifica-se que o modelo de otimização tem capacidade para reduzir o custo operacional por meio do planejamento adequado do funcionamento das bombas no sistema.

Com relação aos valores da confiabilidade, verifica-se, a partir das soluções geradas, que o modelo computacional foi capaz de promover aumento da confiabilidade da rede e também redução das vazões de vazamentos e do custo de energia elétrica, quando comparado com uma regra operacional em que as bombas são mantidas ligadas durante todo o dia.

Para verificar o comportamento das cargas de pressão em cada nó de demanda, foram realizadas simulações hidráulicas com as regras operacionais (NSGAII e SPEA) selecionadas e com "operação extrema" do SDA. Para a hora do sistema em que as pressões se mostravam mais desfavoráveis (12h), os valores da carga de pressão são mostrados na Tabela 5. Verifica-se que as regras operacionais promoveram significativa redução da pressão nodal e garantiram carga de pressão mínima de $10 \mathrm{~m}$, conforme norma brasileira (ABNT, 2017), em todos os nós.

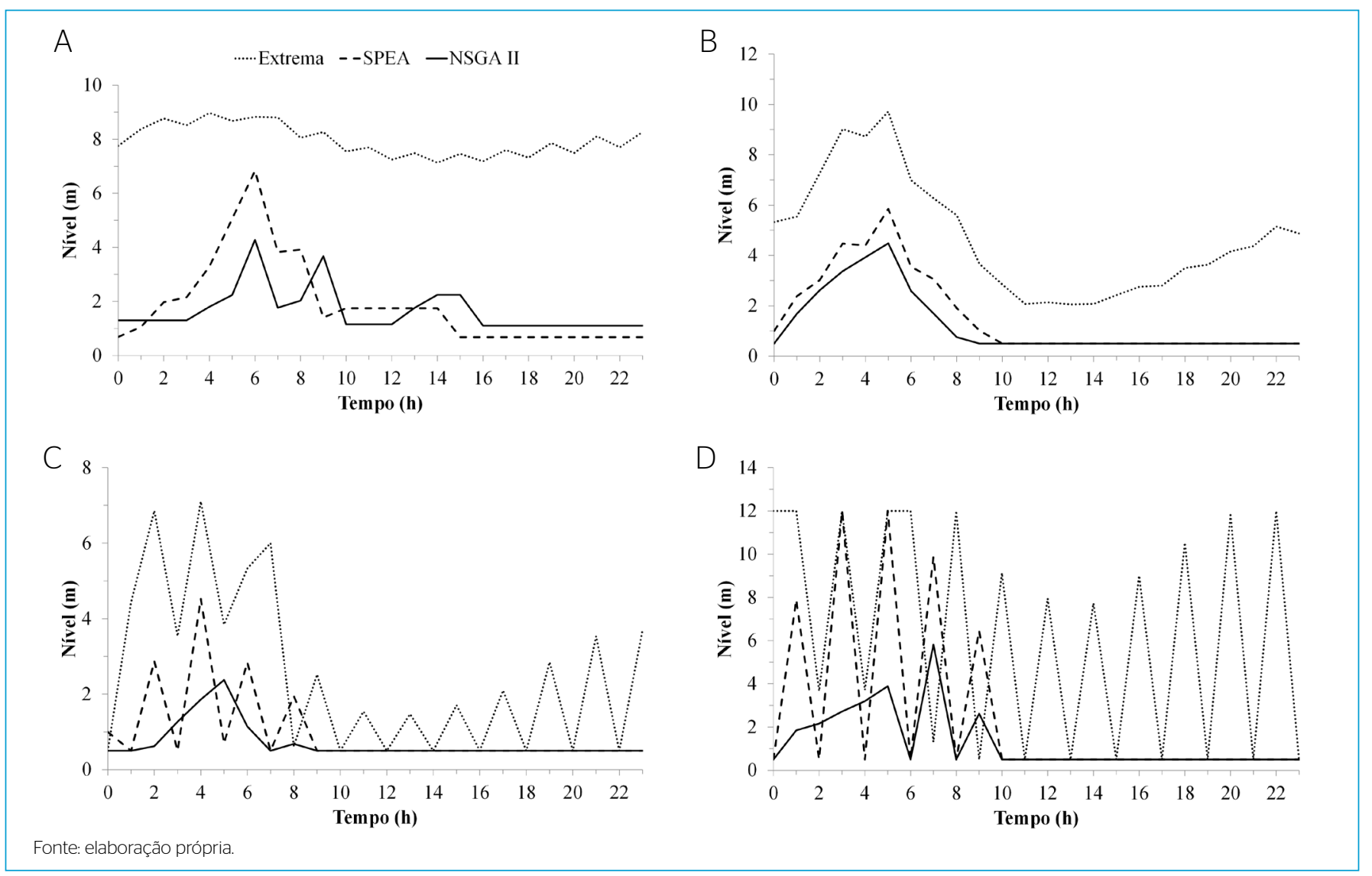

Figura 9 - Variação dos níveis dos reservatórios: (A) a montante da rede de distribuição de água (Tank 10); (B) reservatório 65; (C) reservatório 155; (D) reservatório 165. 
Comparando-se uma condição de "operação extrema" da rede Anytowm, em que todas as bombas são mantidas ligadas, com uma regra operacional do NSGAII, verificou-se que a solução apresentada pelo modelo de otimização promoveu redução de $6,2 \%$ dos vazamentos, redução de $48 \%$ do custo total de energia elétrica e aumento de $10,7 \%$ da confiabilidade do sistema. Ao comparar a condição extrema com uma solução selecionada na frente Pareto gerada pelo algoritmo SPEA, observou-se que houve redução de 5,5\% do volume de vazamentos, redução de $47,2 \%$ do custo total de energia elétrica e aumento de $10 \%$ da confiabilidade do sistema, o que demonstra que o NSGAII foi ligeiramente superior ao SPEA.

\section{CONCLUSÕES}

Um modelo multiobjetivo para a otimização da operação de SDAs foi apresentado neste trabalho. Dois algoritmos evolucionários, SPEA e NSGAII, são comparados e conclui-se que ambos são satisfatórios para serem integrados ao modelo computacional, uma vez que geraram soluções não dominadas para o problema multiobjetivo. As soluções geradas pelo algoritmo NSGAII representam as melhores estratégias operacionais obtidas para a rede de distribuição de água. Entre as soluções geradas pelo algoritmo SPEA, verificou-se que a redução do custo de energia elétrica (isto é, diminuição do bombeamento na rede) não implicou diminuição das perdas por vazamentos, o que demonstra
Tabela 5 - Carga de pressão para a hora mais desfavorável (12 h).

\begin{tabular}{|c|c|c|c|c|}
\hline \multirow[t]{2}{*}{ Hora } & \multirow[t]{2}{*}{ Nó } & Solução NSGAII & Solução SPEA & $\begin{array}{c}\text { Operação ex- } \\
\text { trema }\end{array}$ \\
\hline & & \multicolumn{3}{|c|}{ Carga de pressão $(\mathrm{m})$} \\
\hline \multirow{19}{*}{12} & 20 & 77 & 74 & 82 \\
\hline & 30 & 49 & 54 & 60 \\
\hline & 40 & 41 & 48 & 54 \\
\hline & 50 & 37 & 46 & 51 \\
\hline & 55 & 26 & 36 & 41 \\
\hline & 60 & 43 & 51 & 56 \\
\hline & 70 & 46 & 53 & 58 \\
\hline & 75 & 25 & 36 & 41 \\
\hline & 80 & 38 & 48 & 54 \\
\hline & 90 & 38 & 48 & 54 \\
\hline & 100 & 39 & 51 & 55 \\
\hline & 110 & 45 & 56 & 56 \\
\hline & 115 & 25 & 36 & 40 \\
\hline & 120 & 20 & 33 & 31 \\
\hline & 130 & 20 & 33 & 31 \\
\hline & 140 & 27 & 39 & 43 \\
\hline & 150 & 17 & 29 & 36 \\
\hline & 160 & 20 & 34 & 31 \\
\hline & 170 & 15 & 28 & 29 \\
\hline
\end{tabular}

NSGAll: Non-dominated Sorting Genetic Algorithm; SPEA: Strength Pareto Evolutionary Algorithm.

Fonte: elaboração própria.

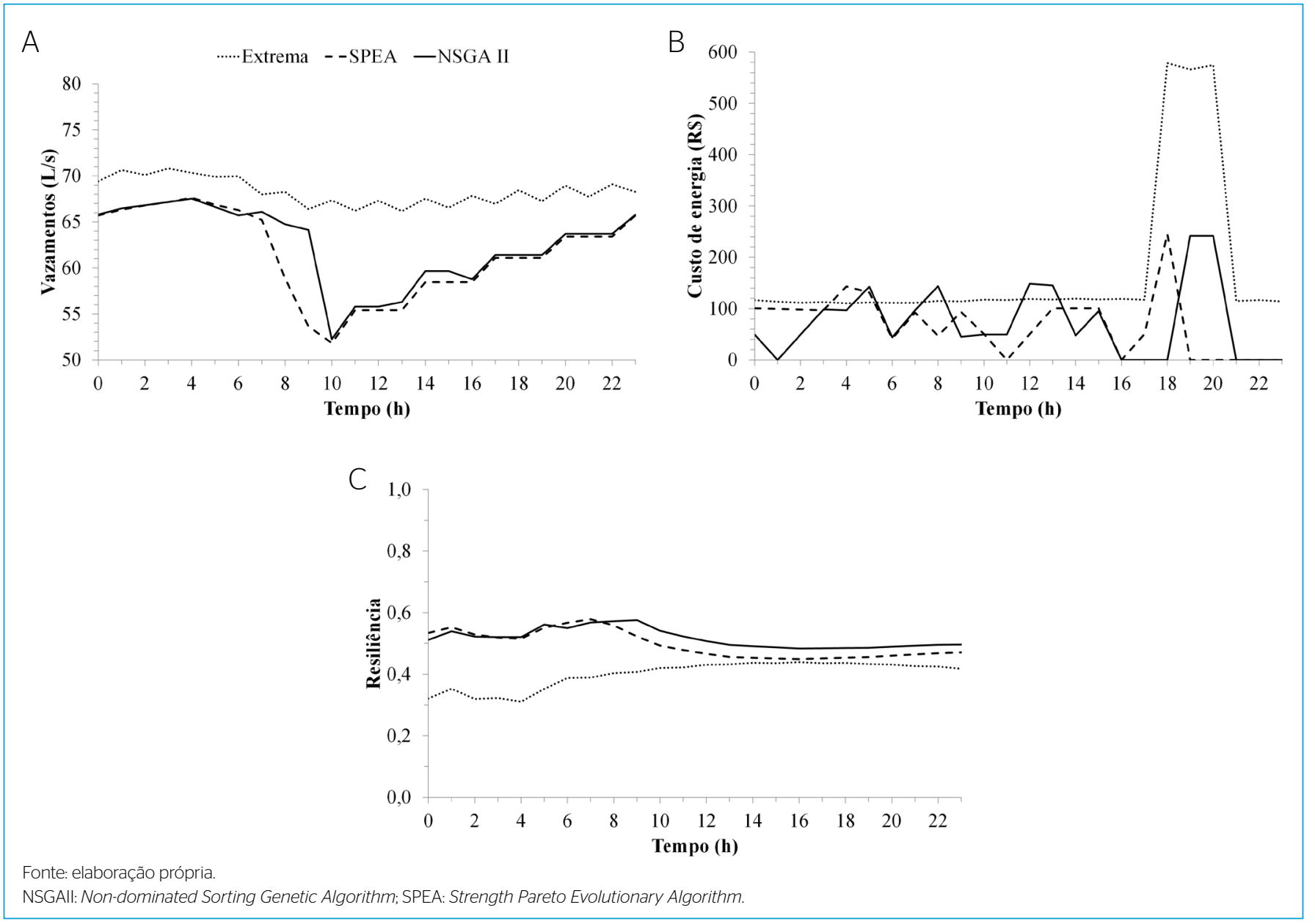

Figura 10 - Variação dos valores das funções objetivo: (A) perdas por vazamentos; (B) custo de energia elétrica no bombeamento; (C) confiabilidade do sistema (função resiliência). 
que tais objetivos podem ser conflitantes para SDAs caracterizados pela presença de reservatórios, conforme afirmado inicialmente por Giustolisi, Laucelli e Berardi (2013).

Recomenda-se o uso do modelo de otimização multiobjetivo para promover a eficiência hidroenergética de SDAs existentes, tanto na macro quanto na microdistribuição, uma vez que as perdas por vazamentos tendem a ser maiores na microdistribuição.

Melhorias do modelo podem ser obtidas com a consideração da localização ótima de válvulas redutoras de pressão, ou mesmo de microturbinas, para promover maior redução de perdas por vazamentos. Quanto ao modelo hidráulico utilizado neste estudo, destaca-se a necessidade da inclusão de demandas dependentes da pressão e de rotação variável das bombas, devido à utilização cada vez maior de inversores de frequência em estações elevatórias.

\section{CONTRIBUIÇÕES DOS AUTORES}

Sousa, A. C.: Conceituação, Curadoria de Dados, Análise Formal, Investigação, Metodologia, Validação, Escrita - Primeira Redação, Escrita - Revisão e Edição. Soares, A. K.: Conceituação, Análise Formal, Obtenção de Financiamento, Administração do Projeto, Recursos, Escrita - Revisão e Edição.

\section{REFERÊNCIAS}

AGÊNCIA NACIONAL DE ENERGIA ELÉTRICA (ANEEL). Resolução no 414. Dispõe sobre as Condições Gerais de Fornecimento de Energia Elétrica. Diário Oficial da União, Brasília, 2010.

ALMANDOZ, J.; CABRERA, E.; ARREGUI, F.; CABRERA JR., E.; COBACHO, $\mathrm{R}$. Leakage assessment through water distribution network simulation. Journal of Water Resources Planning and Management, v. 131, n. 6, p. 458466, 2005. https://doi.org/10.1061/(ASCE)0733-9496(2005)131:6(458)

ALVISI, S.; FRANCHINI, M. Multiobjective Optimization of Rehabilitation and Leakage Detection Scheduling in Water Distribution Systems. Journal of Water Resources Planning and Management, v. 135, n. 6, p. 426-439, 2009. https://doi.org/10.1061/(ASCE)0733-9496(2009)135:6(426)

ASSOCIAÇÃO BRASILEIRA DE NORMAS TÉCNICAS (ABNT). NBR 12218: Projeto de rede de distribuição de água para abastecimento público. Rio de Janeiro: ABNT, 2017

BRANS, J.P.; VINCKE, P.H.; MARESCHAL, B. How to select and how to rank project: The PROMETHEE method. European Journal of Operational Research, v. 24, n. 2, p. 228-238, 1986. https://doi.org/10.1016/0377-2217(86)90044-5

BRILL, E.D.; LIEBMAN, J.C.; LEE, H.L. Optimization of looped water distribution networks. In: TORNO, H.C. (org.). Computer applications in water resources. Nova York: ASCE, 1985. p. 569-571.

BROAD, D.R.; MAIER, H.R.; DANDY, G.C. Optimal Operation of Complex Water Distribution Systems Using Metamodels. Journal of Water Resources Planning and Management, v. 136, n. 4, p. 433-443, 2010. https://doi. org/10.1061/(ASCE)WR.1943-5452.0000052

CAMPISANO, A.; MODICA, C.; VETRANO, L. Calibration of Proportional Controllers for the RTC of Pressures to Reduce Leakage in Water Distribution Networks. Journal of Water Resources Planning and Management, v. 138, n. 4, p. 377-384, 2012. https://doi.org/10.1061/(ASCE)WR.1943-5452.0000197

CARRIJO, I.B. Extração de regras operacionais ótimas de sistemas de distribuição de água através de algoritmos genéticos multiobjetivo e aprendizado de máquina. Tese (Doutorado) - Escola de Engenharia de São Carlos, Universidade de São Paulo, São Carlos, 2004.

CHEUNG, P.B. Análise de reabilitação de redes de distribuição de água para abastecimento via algoritmos genéticos multiobjetivo. 268f. Tese (Doutorado) - Escola de Engenharia de São Carlos, Universidade de São Paulo, São Carlos, 2004.
COLOMBO, A.F.; KARNEY, B.W. Energy and costs of leaky pipes: Toward comprehensive Picture. Journal of Water Resources Planning and Management, v. 128, n. 6, p. 441-450, 2002. https://doi.org/10.1061/ (ASCE)0733-9496(2002)128:6(441)

COLOMBO, A.F; KARNEY, B.W. Impacts of leaks onenergy consumption in pumped systems with storage. Journal of Water Resources Planning and Management, v. 131, n. 2, 2005. https://doi.org/10.1061/(ASCE)0733-9496(2005)131:2(146)

CREACO, E.; PEZZINGA, G. Multiobjective Optimization of Pipe Replacements and Control Valve Installations for Leakage Attenuation in Water Distribution Networks. Journal of Water Resources Planning and Management, v. 141, n. 3, 04014059, 2015. https://doi.org/10.1061/(ASCE) WR.1943-5452.0000458

DEB, K.; AGRAWAL, S: PRATAB, A:; MEYARIVAN, T. A Fast Elitist Non-Dominated Sorting Genetic Algotithm for Multi-Obnjective Optimization: NSGA-Il. In: SCHOENAUER, M.; DEB, K.; RODOLPH, G. (org.). In: PARALLEL PROBLEM SOLVING FROM NATURE CONFERENCE, 6., 2000. Anais [...]. 2000. p. 849-858.

FORMIGA, K.T.M. Otimização Multiobjetivo de Projetos de Redes de Distribuição de Água. 305f. Tese (Doutorado) - Escola de Engenharia de São Carlos, Universidade de São Paulo, São Carlos, 2005.

GESSLER, J. Pipe network optimization by enumeration. In: TORNO, H.C. (org.) Computer applications in water resources. Nova York: ASCE, 1985. p. 572-581.

GIUSTOLISI, O.; LAUCELLI, D.; BERARDI, L. Operational Optimization: Water Losses versus Energy Costs. Journal of Hydraulic Engineering, v. 139, n. 4, p. 410-423, 2013. https://doi.org/10.1061/(ASCE)HY.1943-7900.0000681

GOMES, H.P., CARVALHO, P.S.O. (org.). Manual de Sistemas de Bombeamento: Eficiência Energética. Manual do Usuário. Procel Sanear. João Pessoa: Editora Universitária/UFPB, 2012.

GONÇALVES, R.F. et al. (org.). Conservação de água e energia em sistemas prediais e públicos de abastecimento de água. Rio de Janeiro: ABES, 2009.

INSTITUTO BRASILEIRO DE GEOGRAFIA E ESTATISTICA (IBGE). Sinopse de Censo Demográfico 2010. Rio de Janeiro: IBGE, 2011.

JAMES, K.; CAMPBELL, S.L.; GODLOVE, C.E. Água e energia: aproveitando as oportunidades de eficientização de água e energia não exploradas nos sistemas municipais. Washington, D.C.: Alliance - Aliança para Conservação de Energia, 2002. 
JANNUZZI, P.M. Análise Multicritério e a Decisão em Políticas Públicas: Implementação da Técnica no Aplicativo PRADIN e Aplicações. Rio de Janeiro: Instituto Brasileiro de Geografia e Estatística, 2010.

KUREK, W:; OSTFELD, A. Multiobjective Water Distribution Systems Control of Pumping Cost, Water Quality, and Storage-Reliability Constraints. Journal of Water Resources Planning and Management, v. 140, n. 2, p. 184-193, 2014. https://doi.org/10.1061/(ASCE)WR.1943-5452.0000309

LANSEY, K.; MAYS, L. A methodology for optimal network design. In: TORNO, H.C. (org.). Computer applications in water resources. Nova York: ASCE, 1985. p. 732-738.

MALA-JETMAROVA, H.; BARTON, A.; BAGIROV, A. Exploration of the TradeOffs between Water Quality and Pumping Costs in Optimal Operation of Regional Multiquality Water Distribution Systems. Journal of Water Resources Planning and Management, v. 141, n. 6, 04014077, 2015. https:// doi.org/10.1061/(ASCE)WR.1943-5452.0000472

MORGAN, D. R.; GOULTER, I.C. Water distribution design with multiple demands. In: TORNO, H.C. (org.). Computer applications in water resources. Nova York: ASCE, 1985. p. 582-590.

NICOLINI, M.; GIACOMELLO, C.; DEB, K. Calibration and Optimal Leakage Management for a Real Water Distribution Network. Journal of Water Resources Planning and Management, v. 137, n. 1, p. 134-142, 2011. https://doi. org/10.1061/(ASCE)WR.1943-5452.0000087

ODAN, F.K.; REIS, L.F.R.; KAPELAN, Z. Real-Time Multiobjective Optimization of Operation of Water Supply Systems. Journal of Water Resources Planning and Management, v. 141, n. 9, 04015011, 2015. https://doi.org/10.1061/(ASCE) WR.1943-5452.0000515

ORMSBEE, L. Opnet: A Nonlinear Design Algorithm For Hydraulic Networks. In: TORNO, H.C. (org.). Computer applications in water resources. Nova York: ASCE, 1985. p. 739-748.
PINO, E.; VALLE, A.; CONDORI, F.; MEJIA, J.; CHAVARRI, E.; ALFARO, L. Diseño Óptimo de Redes de Distribución de Agua Usando Un Software Basado En Microalgoritmos Genéticos Multiobjetivos. Ribagua, v. 4, n. 1, p. 6-23, 2017. https://doi.org/10.1080/23863781.2017.1317087

PUDAR, R.S.; LIGGETT, J.A. Optimization of Looped Water Distribution Systems. Journal of the Hydraulics Division, v. 103, n. HY3, p. 281-294, 1992.

ROSSMAN, L. EPANET 2 users manual. Cincinnati: U.S. Environmental Protection Agency, 2000.

SISTEMA NACIONAL DE INFORMAÇÕES SOBRE SANEAMENTO (SNIS). Diagnóstico dos serviços de água e esgotos: 2018. Brasilia: MCIDADES, SNSA, 2018.

SRINIVAS, N.; DEB, K. Multiobjective Optimazation Using Nondominated Sorting in Genetic Algorithms. Evolutionary Computation, v. 2, n. 3, p. 221248, 1994. https://doi.org/10.1162/evco.1994.2.3.221

TODINI, E. Looped Water Distribution Networks Design Using a Resilience Index Based Heuristic Approach. Urban Water, v. 2, n. 2, p. 115-122, 2000 https://doi.org/10.1016/S1462-0758(00)00049-2

WALSKI, T.M.; BRILL JR., E.D; GESSLER, J; GOULTER, I.C; JEPPSON, R.M. LANSEY, K; LEE, H.L.; LIEBMAN, J.C; MAYS, L; MORGAN, D.R;; ORMSBEE, L. Battle of networks models: Epilogue. Journal of Water Resources Planning and Management, v. 113, n. 2. p. 191-203, 1987. https://doi.org/10.1061/(ASCE)0733-9496(1987)113:2(191)

WU, W: SIMPSON, A.R; MAIER, H.R; MARCHI A. Incorporation of Variable-Speed Pumping in Multiobjective Genetic Algorithm Optimization of the Design of Water Transmission Systems. Journal of Water Resources Planning and Management, v. 138, n. 5, p. 543-552, 2012. https://doi.org/10.1061/(ASCE)WR.1943-5452.0000195

ZITZLER, E; THIELE, L. Multiobjective optimization using evolutionary algorithms a comparative case study. In: EIBEN, A.E; BACK, T:; SCHOENAUER, M:; SCHWEFEL, H.P. (org.) INTERNATIONAL CONFERENCE ON PARALLEL PROBLEM SOLVING FROM NATURE, 5., 1998. Anais [...]. Berlim: Springer, 1998. p. 292-301. 